\title{
THE MOBILITY OF ELECTRONS IN PURE NITROGEN.
}

\author{
By LeONARD B. LOEB.
}

SYNOPSIS.

\begin{abstract}
Mobility of electrons in pure nitrogen was determined, using the Rutherford alternating potential method, for pressures ranging from 75 to $600 \mathrm{~mm}$., for frequencies ranging from 7,000 to 150,000 cycles (obtained from an audion oscillating circuit), and with electric fields of from Io to 100 volts $/ \mathrm{cm}$. Reduced to atmospheric pressure the mobility found is of the order of $10,000 \cdot \mathrm{cm}$. sec.,/many times the highest value previously obtained, and it was observed to vary with pressure and electric field according to the equation $K=57 \mathrm{I}, 000 /(2 \mathrm{I}+760 \mathrm{~V} / p d)$, where $V / d$ is in volts $/ \mathrm{cm}$. and $p$ in $\mathrm{mm}$. of $\mathrm{Hg}$. A discussion of the possible sources of error shows that none can be responsible for this variation with $V / p d$, and the form of the mobility curves confirms this variation. A theoretical interpretation of these results on the basis of the Townsend equation for electron mobility leads to the conclusions: that if the Townsend theory is correct (I) either the energy lost at each impact of an electron with a nitrogen molecule or the mean free path must be a function of $V / p d$, and (2) in either case the mean free path for velocities of the normal agitation must be about 22 times the mean free path of the gas molecules instead of $4 \sqrt{2}$ times, as given by the kinetic theory.
\end{abstract}

\section{Introduction and Previous Results.}

THE results obtained in a study of the attachment of electrons to neutral molecules to form negative ions, ${ }^{1}$ indicated that the mobility constant for the electrons in a gas at atmospheric pressures was apparently not constant. It thus became of interest to determine the mobility of electrons in gases in which they move unattached to molecules at atmospheric pressures in order to discover what conditions govern the variations of mobility. The mobilities of electrons in pure argon and nitrogen at atmospheric pressures were first determined by Franck, ${ }^{2}$ who obtained $206 \mathrm{~cm}$. $/ \mathrm{sec}$. for the highest value in the former and $145 \mathrm{~cm}$. $/ \mathrm{sec}$. for the highest value in the latter gas. Haines ${ }^{3}$ measured the mobilities of electrons in nitrogen and found mobilities as high as $506 \mathrm{~cm}$./sec. while the average value obtained lay at about $367 \mathrm{~cm}$. $/ \mathrm{sec}$. In hydrogen he found the highest value to be $170 \mathrm{~cm} . / \mathrm{sec}$. Finally

${ }^{1}$ Loeb, L. B., Phys. Rev., Vol. XVII., No. 2, pp. I06-I07, Feb., I921.

${ }^{2}$ Franck, J., Verh. d. Deut., Phys. Ges., I2, p. 613, I9г3.

${ }^{3}$ Haines, Phil. Mag., 6, 30, p. 503, I9I5. 
Wellisch ${ }^{1}$ working with what he thought were purely electronic carriers in $\mathrm{CO}_{2}$ obtained mobilities which varied from $256 \mathrm{~cm}$./sec. to much lower values. In $\mathrm{H}_{2}$ he got a value of $\mathbf{I}, 700 \mathrm{~cm}$. $/ \mathrm{sec}$. for the mobility.

\section{Experimental Method.}

In the present work the mobilities were determined on the photoelectrons liberated from a brass plate $(P)$, Fig. I, struck at grazing incidence by ultraviolet light from a quartz mercury arc, using the Rutherford alternating current method. The experimental method of making the determinations was essentially the same as that used by the writer in an earlier ${ }^{1}$ paper on negative ions. The gas used was pure nitrogen in which it has been shown that the electrons do not attach to form ${ }^{2}$ ions. $^{5}$ The method of purification was carried out as described in the paper ${ }^{5}$ showing this to be the case. A reduction of the contamination of the gas while in the measuring chamber by vapors arising from sealing waxes and organic materials used in closing the chamber was accomplished by the use of a new ionization chamber. It was similar to the one previously described, except that the rubber gasket was replaced by a ground steel joint with a mercury seal.

The mobilities were measured by the use of the high-frequency oscillations from two vacuum tube oscillators of the Western Electric "E" tube type operating in parallel. The circuits used are shown in the diagram, Fig. I. In some cases the oscillations were taken directly off the primary condenser, and led to the plate $P$ of the measuring system. In many others they were taken from the condenser of a secondary circuit closely coupled to the primary circuit, and tuned to resonance with it. The alternating potential was varied in the measurements by changing the resistance $(r)$ in the plate circuit of the oscillators. This change in the resistance resulted in a gradual change in the frequency with the voltage, sometimes causing a reduction in frequency as the voltage decreased and at other times causing an increase, depending on the constants of the oscillating circuit used at the time. It therefore became necessary to measure the frequency of the oscillations at several values of the voltage in order to obtain accurate values of the mobility. This was done with a wave meter. The frequencies thus obtained appeared to be in good accord with those computed from the circuit constants, where computation was possible. The alternating potentials were measured with Kelvin type static voltmeters which were calibrated from time to time against a standard Weston voltmeter. A large number

${ }^{1}$ Wellisch, E. M., Am. Jour. Science, July, I9r 5.

${ }^{2}$ Loeb, L. B., Proc. Nat. Acad. Sciences, 6, No. 7, pp. 335-44I, July, 1920. 
of mobility curves were taken covering frequencies ranging from 30,000 cycles up to 150,000 cycles per second, and pressures of nitrogen from $600 \mathrm{~mm}$. to $75 \mathrm{~mm}$. of $\mathrm{Hg}$. It was found possible to study the mobility curves with potentials which varied from 20 volts to 140 in all cases, and from 30 volts to 300 in a few cases. Below 20 volts it was not satisfactory to make mobility measurements as the oscillations became rather unsteady, though some determinations were carried down to less than ten volts. The plate distances $P E$ used in the measuring chamber were $2 \mathrm{~cm}$. and $\mathrm{I} .5 \mathrm{~cm}$.

\section{Results.}

In Figs. 2 and 3 are given some of the typical mobility curves, obtained

\begin{tabular}{|c|c|c|c|c|c|}
\hline No. & $\begin{array}{c}\text { Press. } \\
\text { in } \mathbf{M m} . \\
p .\end{array}$ & $\begin{array}{c}\text { Frequency } \\
n .\end{array}$ & $\begin{array}{c}\text { Intercept } \\
\text { Corrected } \\
V_{0}\end{array}$ & $\underset{u .}{\text { Mobility }}$ & $\begin{array}{c}\text { Mobility } \\
\text { Constant } \\
K .\end{array}$ \\
\hline $1 \ldots \ldots$ & 610 & $28,000 ?$ & 7.6 & 34,800 & 28,000 \\
\hline $2 \ldots$ & 597 & 31,800 & 14.3 & 21,000 & 16,500 \\
\hline $3 \ldots$ & 607 & 49,200 & 24.8 & 17,350 & 13,850 \\
\hline $4 \ldots$ & 599 & 53,100 & 28.5 & 17,600 & 13,700 \\
\hline 5. & 615 & 62,200 & 32.6 & 17,900 & 14,600 \\
\hline 6. & 606 & 68,000 & 40.1 & 14,850 & 11,850 \\
\hline $7 \ldots$ & 604 & 76,000 & 57.3 & 11,600 & 9,230 \\
\hline 8. . & 599 & 75,000 & 66.8 & 10,650 & 8,400 \\
\hline 9. & 625 & 95,500 & 80.1 & 11,100 & 9,200 \\
\hline 10 . & 603 & 82,200 & 90.7 & 8,600 & 6,800 \\
\hline $11 \ldots$ & 597 & 88,000 & 94.0 & 8,200 & 6,440 \\
\hline
\end{tabular}

\begin{tabular}{|c|c|c|c|c|c|}
\hline No. & $\begin{array}{c}\text { Press. } \\
\text { in } \underset{p m}{\mathrm{Mm} .}\end{array}$ & $\begin{array}{c}\text { Frequency } \\
n .\end{array}$ & $\begin{array}{c}\text { Intercept } \\
\text { Corrected } \\
V_{0}\end{array}$ & $\begin{array}{c}\text { Mobility } \\
u .\end{array}$ & $\begin{array}{c}\text { Mobility } \\
\text { Constant } \\
K .\end{array}$ \\
\hline $1 \ldots \ldots \ldots$ & 302 & 82,000 & 38.7 & 18,000 & 7,340 \\
\hline $2 \ldots$ & 303 & 98,000 & 74.4 & 11,500 & 4,610 \\
\hline $3 \ldots$ & 166 & 93,000 & 32.4 & 25,100 & 5,500 \\
\hline $4 \ldots$ & 166 & 115,000 & 104.3 & 9,650 & 2,100 \\
\hline $5 \ldots$ & 75 & 141,000 & 75.4 & 16,250 & 1,610 \\
\hline 6. & 74.5 & 118,000 & 48.6 & 21,200 & 2,090 \\
\hline $7 \ldots$ & 75.0 & 91,000 & 21.0 & 38,000 & 3,750 \\
\hline
\end{tabular}

by plotting the electrometer current against the electrostatic voltmeter reading under the conditions of frequency and pressures indicated in the legend. The intercepts of these curves with the voltage axis (or in the case of the curves with markedly asymptotic feet the intercepts of the extrapolated straight portions of the curves with the axis) yield the value of $V_{0}$ from which the mobility constant of the electron $K$ is determined by the equation, 
Vol. XIX.] MOBILITY OF ELECTRONS IN PURE NITROGEN.
No. 1.

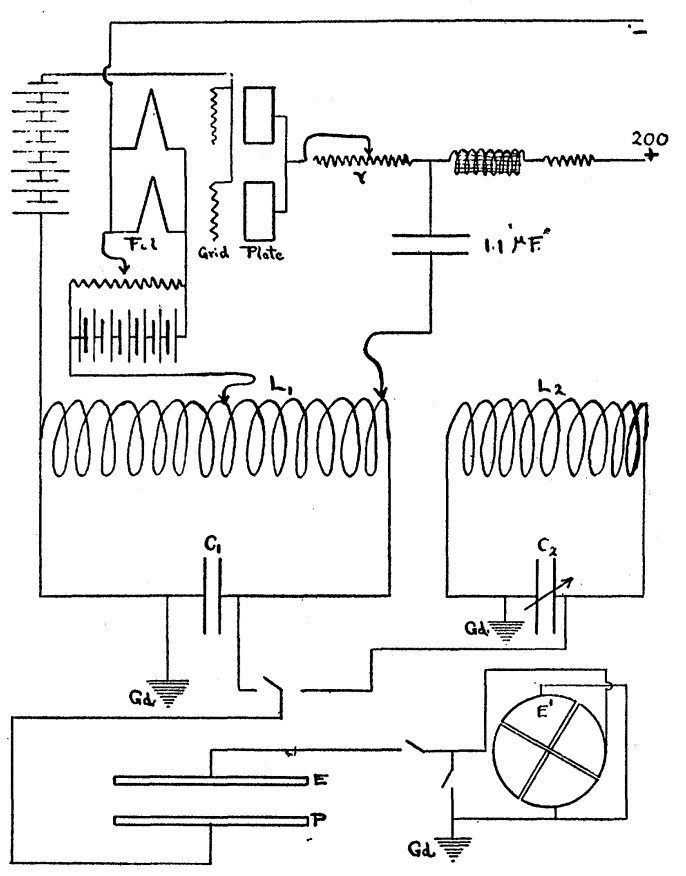

Fig. 1.

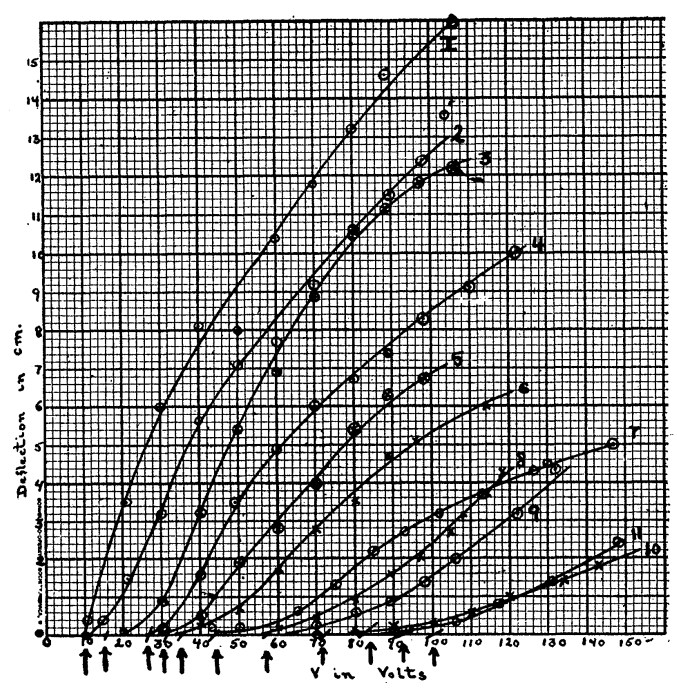

Fig. 2. 


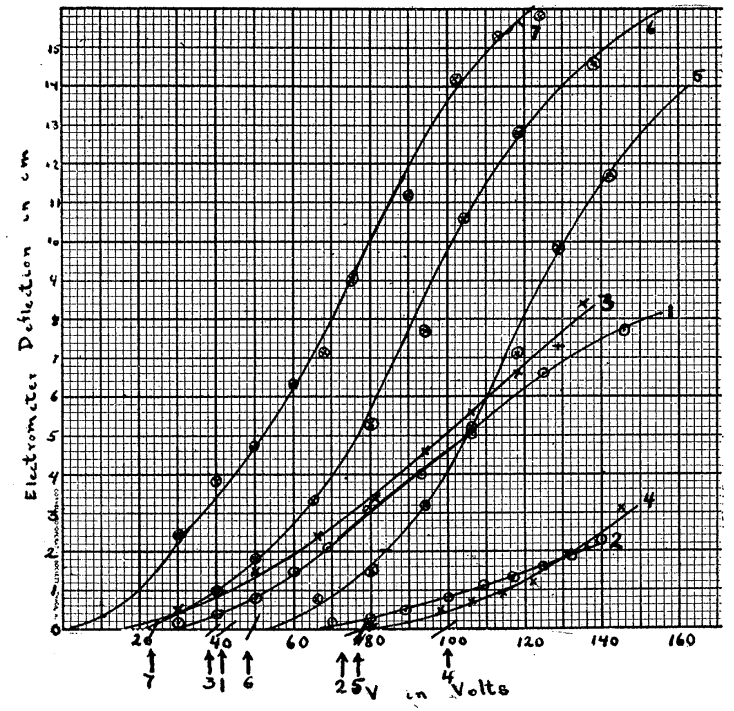

Fig. 3 .

$$
K=\frac{\pi n d^{2} p}{\sqrt{2} V_{0760}}
$$

where $n$ is the frequency of the oscillation, $d$ is the distance between the plates $P E$, and $p$ is the pressure in $\mathrm{mm}$. of $\mathrm{Hg}$. In this case as before the value of $V_{0}$ as obtained from the intercept must be corrected by a deduction of 4.6 per cent. of its value in the case where $d=2 \mathrm{~cm}$. to take care of the drop in potential across the electrometer while the readings were being made. A discussion of the sources of error and the nature of the curves obtained will be postponed until a later point in the paper.

The mobility constants thus obtained were of the order of magnitude of $10,000 \mathrm{~cm} . / \mathrm{sec}$. volt $/ \mathrm{cm}$. This is a value far in excess of any obtained by the previous investigators. It seems likely that the successful measurement of such high values was due to three essential differences between the wiiter's mode of procedure and those of the previous observers. All three of the workers mentioned above used electrons generated by the action of alpha particles on the gas in an auxiliary chamber (i.e., they used the Franck modification of the Rutherford method), while the writer used photoelectrons freshly liberated from one of the plates. Furthermore the writer used extreme measures not only for purifying his nitrogen gas but also for reducing the contamination from the chamber to a minimum. That this was successful was shown by the fact that 
Vol. XIX. ] MOBILITY OF ELECTRONS IN PURE NITROGEN.
No. I.

nitrogen kept in the chamber for as long as Io days still yielded electron mobilities which were of the same value as when the gas was freshly prepared. Finally the use of very high frequencies by the writer enabled only the purely electronic carriers to be studied. The other workers had used frequencies at most as high as 3,000 cycles per second, while the writer never used frequencies below 7,000 cycles and generally worked above 20,000 cycles. The mobility constants were furthermore found to vary through wide limits. It was found that by plotting $K$ the mobility constant obtained from the equation above for a given value of $d$ and $p$ as a function of $V_{0}$, the voltage intercept, a smooth curve could in general be drawn through the points. For each value of $d$ or $p$ a

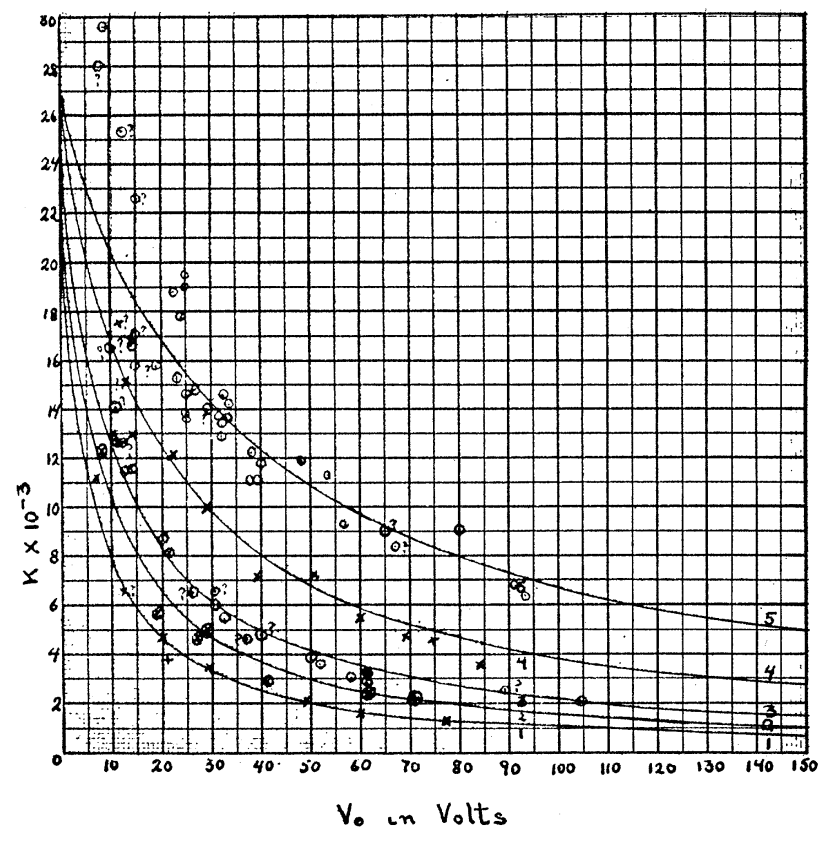

Fig. 4.

\begin{tabular}{l|c|c}
\multicolumn{3}{c}{ Mobility Constant $K$ Plotted against $V_{0}$ Corrected. } \\
\hline \multicolumn{1}{c|}{ No. } & $\begin{array}{c}\text { Press. } p \\
\text { in Mm. }\end{array}$ & $\begin{array}{c}\text { Plate Distance } \\
d \text { in Cm. }\end{array}$ \\
\hline $1 \ldots \ldots \ldots \ldots \ldots \ldots \ldots \ldots \ldots$ & 75.5 & 2.00 \\
$2 \ldots \ldots \ldots \ldots \ldots \ldots \ldots \ldots \ldots$ & 160.0 & 2.00 \\
$3 \ldots \ldots \ldots \ldots \ldots \ldots \ldots \ldots \ldots$ & 304.0 & 2.00 \\
$4 \ldots \ldots \ldots \ldots \ldots \ldots \ldots \ldots \ldots$ & 150.0 & 1.52 \\
$5 \ldots \ldots \ldots \ldots \ldots \ldots \ldots \ldots$ & 606.0 & 2.00 \\
\hline
\end{tabular}

different curve was obtained. The curves obtained for all the determinations are shown in Fig. 4. The legend gives the conditions under 
which they were obtained. The curves were hyperbola of the form $K=a /(b+V)$. Furthermore if the equation for one curve with a given $d^{\prime}$ and $p^{\prime}$ be obtained (i.e., that for curve (I) at $d=2 \mathrm{~cm}$. and $p=75 \mathrm{~mm}$. which is $K=\mathrm{II} 3 /(4 . \mathrm{I} 8+V))$ then it will be found that the equation for any of the other curves may be obtained from it, by multiplying the constants of this equation by $d p / d^{\prime} p^{\prime}$. Where $d^{\prime}$ and $p^{\prime}$ are the values of pressure and distance corresponding to the curve whose equation is known, and $d$ and $p$ are the pressure and distance corresponding to the curve to be determined. In other words, all the results may be expressed by means of the equation

$$
K=\frac{57 \mathrm{I}, 000}{2 \mathrm{I}+(760 V) / p d},
$$

within the limits of error of the measurements. That this is the case is seen from the fit of the observed points to the curves plotted from the above formula. In noting this agreement it is well to point out that too much reliance must not be placed on the values of $K$ obtained for intercepts at values of $V_{0}$ less than ro volts. At this point owing to the extrapolation necessary to get the intercepts of the mobility curves, the values of $K$ can easily vary as much as 30 per cent. or more. It is also interesting to note that as the pressures increase the deviations of the points on both sides of the theoretical curves become greater. This is in part due to the fact that a variation of say Io per cent. from the curve at the higher values of the mobility will actually be greater in magnitude, than in the case where the mobilities are low.

The result obtained seems quite surprising and it is natural to inquire into the effects of possible sources of error before accepting them.

\section{SOURCES OF ERror.}

(a) Effect of Wave Form.-The first question which arises is as to whether changes in the wave form of the oscillations due to higher harmonics might not influence the results. The equation given for the mobility for the case of an oscillation of pure sine-wave form was $K=2.22 n d^{2} / V_{0}$. For a square wave form, the equation becomes $K=2 n d^{2} / V_{0}$. The error introduced by a change from a sine-wave to a square wave, an extreme case, would be to give values of the mobility which are II per cent. too high. Errors due to neglect of wave form will therefore in general be slight.

(b) How Does the Variation of $K$ with $V$ Affect the Mobility Curves and Hence these Determinations? - One may next inquire as to the effect of the variation of the mobility constant $K$ with the voltage on the 
shape of the mobility curves. A theoretical study of the shape of the mobility curve for photoelectrons, liberated while the alternating field is acting, assuming a square wave form and that $K$ is a constant yields the equation $I / I_{0}=\left(V-V_{0}\right) / V$; where $I$ is the current, $I_{0}$ is the maximum possible current, and $V_{0}$ is the critical value of the voltage when the electrons can just get to $E$. It has been found that for the case of the negative ions, where $K$ is constant, such an equation reproduces the form of the observed mobility curves with a square wave fairly well. For the case of a sine-wave the equation for the electronic current as a function of the voltage for $K$ constant is found to be $I / I_{0}=\mathrm{I} / \pi \cos ^{-1}\left\{\left(2 V_{0} / V\right)-\mathrm{I}\right\}$. The two types of curves for two different values of $V_{0}$ are shown in Fig. 5 .

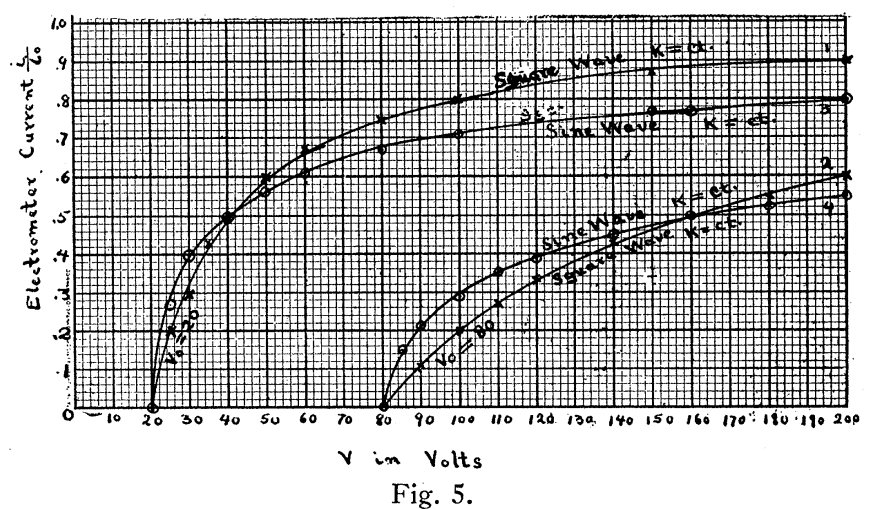

Theoretically computed curves for variation of current with an alternating voltage for ions or electrons for which the mobility constant $K$ is constant. This is computed for photoelectrons.

\begin{tabular}{|c|c|c|}
\hline No. & Wave Form. & $V_{0}$. \\
\hline $\begin{array}{l}1 \ldots \ldots \ldots \ldots \ldots \ldots \\
2 \ldots \ldots \ldots \ldots \ldots \ldots \ldots \ldots \ldots \ldots \\
3 \ldots \ldots \ldots \ldots \ldots \ldots \ldots \ldots \ldots \ldots\end{array}$ & $\begin{array}{l}\text { square } \\
\text { square } \\
\text { sine } \\
\text { sine }\end{array}$ & $\begin{array}{l}20.0 \\
80.0 \\
20.0 \\
80.0\end{array}$ \\
\hline
\end{tabular}

It is seen that they are similar in general form except that the curve for the sine-wave rises a bit more abruptly at first. If now $K$ varies, as was found experimentally to be the case, viz., $K=a /(b+V)$, the equation for the square wave becomes one of the form,

$$
I / I_{0}=\left(\mathrm{I}-\frac{V_{0}+V / b}{V}\right),
$$

where $V_{0}=n d^{2} / K_{0}$ and $K_{0}=a / b$. On plotting this equation we get the types of curves seen in Fig. 6 for the positive values of the current. 
The value of the intercept will if substituted in the mobility equation for a square wave form yield (e.g., $\left.K=2 n d^{2} / V_{0}\right)$ values of $K$ that vary as $K$ was assumed to vary. For the case of the sine-wave with $K=a /(b+V)$ it is impossible to determine the equation directly. The mobility curves may, however, be determined for a given set of conditions, by means of graphic integration. This was done, and the curves so obtained for three values of $V_{0}$ are shown in Fig. 6. These curves are not unlike those obtained for the square wave form and differ from them chiefly in the sharper rise of the curve near $V_{0}$ and a slightly lower value of



Fig. 6.

Theoretical curves for variation of current with an alternating voltage for electrons, for which $K$ is a variable with $V$ according to the equation, $K=a /(b+V)$. Values of the constants taken from experiment. Curves $I, 2$ and 3 correspond to the curves for a square wave under the conditions of the experiment at frequencies of $30,000,50,000$ and 70,000 cycles and $606 \mathrm{~mm}$. pressure. Curves 4,5 and 6 correspond to the curves with a sine wave form and the same frequencies respectively at a pressure of $760 \mathrm{~mm}$. The plate distance here is $2 \mathrm{~cm}$.

$i / i_{0}$. It is at once seen that the apparent saturation values of the curves of Fig. 6 do not nearly rise to the values attained with $K$ a constant (see Fig. 5). It is also to be noticed that these saturation values are progressively lower the greater the value of $V_{0}$. This peculiarity which had been observed in all the experimental curves (see Figs. 2 and 3 ), had puzzled the writer for some time, until it was found to be a natural consequence of the variation of $K$ with voltage.

(c) Discussion of the Asymptotic Feet of the Mobility Curves.-There is another peculiarity of the curves which up to the present has not been satisfactorily explained. Since the electrons in crossing the plates make millions of collisions before they cross, it is to be expected that they all move with a nearly uniform velocity of drift in the direction of the field. Thus the intercepts of the mobility curves with the voltage axis should be quite sharp. Actually this is not the case. The mobility curves 
have asymptotic feet which become more pronounced as the value of $V_{0}$ for the curves increases and so drags out these portions of the curves over a greater range of voltages. A study of these feet indicate that they are due to a factor which increases the electrometer current more and more rapidly as the quantity $V_{0}-V$ decreases to 0 . This portion of the curves is also apparently increased in importance by a decrease in pressure or a decrease in the distance between the plates. At present the only plausible explanation of these feet is that they are due to the formation of negative ions in the gas. Now the gas used, though quite pure, is not absolutely so. Therefore while the electrons are oscillating back and forth in the space between the plates some of them attach to form negative ions. These, owing to their low mobilities, remain between the plates. In the course of the million cycles which elapse while a measurement is being made this process might build up such a gradient of ions that some of these will diffuse to the upper plate $E$ and register a current. The gradient built up will be the sharper (and hence the number diffusing will be the greater), the nearer the electrons get to crossing the distance between the plates, i.e., the smaller $\left(V_{0}-V\right)$, and the smaller the distance $d$. As a decrease in the pressure increases the coefficient of diffusion of the ions, it would also increase the number that get across. An attempt was made to remove these feet by putting a weak but constant field in the circuit in such a manner as to remove the accumulated ions as they were formed. The reduction in prominence of the feet produced by a field of .75 volt per $\mathrm{cm}$. was however not sufficiently great to permit the explanation offered to account for the whole effect. It does however account for a portion of it.

(d) A Possible Effect of the Unsymmetry of the Waves Relative to the Ground Potential.-Finally one must consider the possibility that a state of affairs exists in the oscillating system such that the oscillations, that is the positive and negative crests of the waves, are unsymmetrically placed with respect to the ground potential. This condition, though unlikely in this type of circuit is, however, important enough when present to merit a brief discussion. Consideration will show that when the negative side of the wave has a higher peak value than the positive side, by even a small fraction, it will become impossible to obtain any mobility curves at all. For the electrons will be accelerated by the field for a longer time interval and by a greater average force when the phase is negative than when it is positive. Accordingly the electrons advance towards the electrometer plate $E$ a distance $d^{\prime}$, and are pulled back from it a distance equal to $d^{\prime \prime}$, where $d^{\prime}>d^{\prime \prime}$. Thus since the measurements last over a million or more cycles the electrons 
get across the plates for very small potential differences. With the positive peaks higher than the negative peaks, the ions will cross as in the case for symmetrical waves. However, the value of $V_{0}$ taken from the curves, as registered on the static voltmeter, will be higher than the actual value of the driving potential. Also the time over which the potential acts will be shorter than that assumed from the frequency. The mobility as computed from readings under these conditions, will be lower than the actual value. If such an unsymmetry should actually occur and increase systematically with the increase in frequency, it might produce results analogous to those obtained. Calculation shows that to produce a mobility which is but 78 per cent. of the true value it requires that the height of the negative peak be .82 that of the positive peak. If the mobility were to be reduced to one fifth its true value the negative peak would have to be .33 as high as the positive peak. With a Braun tube oscillograph no unsymmetry of the oscillations over a considerable range of frequencies could be detected, within the limits of resolution of the oscillograph used. The instrument could easily have shown unsymmetry of .82 between the two peaks. Other tests also failed to reveal any unsymmetry. In no case was unsymmetry with the negative side greater than the positive side manifested by any mobility measurements. Finally the diversity of coils and the diversity of connections used in obtaining the oscillations should a priori have caused no systematic change of any unsymmetry present such as would be required to yield the results described.

\section{The Interpretation of the Results on the Basis of Townsend's Electron Mobility Equation.}

(a) Modification of the Langevin Equation by Townsend.-An interpretation of the variation of the mobility found is difficult. Townsend ${ }^{6}$ suggests that the Langevin ${ }^{7}$ equation for ionic mobilities may be applied to the case of electrons. In doing this one must assume that owing to the elastic impacts which the electron makes with the more massive molecules its energy of agitation in the gas (i.e., its temperature), increases as it moves through the gas, due to the action of the field. Townsend ${ }^{8}$ gives the Langevin equation, when it has been reduced to the case of a body of mass small compared with that of the molecules as

$$
K=.8 \mathrm{I} 5 \frac{e}{m} \frac{\lambda}{\bar{c}}
$$

where $e$ is the electronic charge, $m$ its mass, $\lambda$ its mean free path, and

${ }^{6}$ Townsend, J. S., "Electricity in Gases," Oxford, I9I4, p. I74 ff; Phil. Mag., Vol. 40, I920.

${ }^{7}$ Langevin, P., Ann. de Chimie et de Phys., 8, 5, p. 245, I905.

8 Townsend, J. S., Electriçity in Gases, p. I80. 
$\bar{c}$ its mean velocity of thermal agitation. This equation must be modified according to Townsend by multiplying the $\bar{c}$ by a quantity $\sqrt{k}$ before it may be applied to the case of the electron in an electric field. (A similar equation is given by Lenard ${ }^{12}$ except that in his equation the constant term is .5 instead of .815 and that he uses $\alpha_{1}$ in place of $\sqrt{k .)}$ This quantity $\sqrt{k}$ must then be a quantity of such a form that when it is multiplied by $c$ it gives the average velocity of agitation of the electron in the field. If the maximum energy which the electron can acquire due to the field alone be called $W$, the average energy of the electron as a result of the heat motions and the field must be given by $\left[2\left(m \bar{c}^{2} / 2\right)+W\right] / 2$. The average velocity of the electron as a result of thermal agitation and the action of the field will then become $\sqrt{c^{2}+W / m}$, and hence the quantity $\sqrt{k}=\sqrt{\mathrm{I}+W / m \bar{c}^{2}}$.

(b) Consequences of This Equation for Elastic Impacts. - Now the value of $W$ will depend on how the electron gains and loses energy in the field. If, as is the case for the most part in helium and the monatomic gases. the impacts are nearly perfectly elastic, it will according to the quantum theory continue to gain in energy of agitation until it acquires enough energy to suffer an inelastic resonance impact. At the high pressures under which the experiments above were conducted the electrons probably exceeded their resonance potentials $R$ very little before suffering a resonance impact. Also if the total fall of potential between the plates was several times that of the resonance potential, the value of $W$ was probably close to $R \times e$, where $e$ is the electronic charge. Thus the average velocity of the electrons in this case would approximate a value of the form $\bar{c} \sqrt{\mathrm{I}+R e / m \vec{c}^{2}}$. The mobility of the electron on Townsend's assumptions would then be given by

Since $\operatorname{Re} / m \bar{c}^{2}>>$ I,

$$
K=.8 \mathrm{I} 5 \frac{e}{m \frac{\mathrm{a}}{c} \sqrt{\mathrm{I}+\frac{R e}{m \bar{c}^{2}}}},
$$

$$
K=.8 \mathrm{I} 5 \frac{e}{m} \frac{\lambda}{\bar{c} \sqrt{\frac{R e}{\bar{c}^{2}}}} \cdot
$$

It is seen at once that this mobility would be a constant depending solely on $R$, and would not vary as a function of $p$ or $d$ as was experimentally found to be the case.

(c) Consequences of this Equation for the Case of Partially Elastic Impacts.-Thus one must conclude that the electron in nitrogen does not 
behave as in helium, but that it loses a fraction of its energy $(f)$ at each impact. If this is the case Compton ${ }^{12}$ has shown that the value of $W$ for the electrons, after a sufficient number of impacts, approaches a terminal value given by

$$
W=U e=.575 \frac{V e \lambda}{d \sqrt{f}}
$$

which is in agreement with a result arrived at by Pidduck in a paper entitled "The Abnormal Energy of an Electron in a Gas." " 11 Here $V$ is the potential across the plates, $d$ is the distance between them, and $f$ is the fraction of the energy of the electron lost at each impact. The value of $\sqrt{k}$ then becomes

and hence

$$
\sqrt{k}=\sqrt{\mathrm{I}+.575 \frac{V e \lambda}{\sqrt{f} d m \bar{c}^{2}}},
$$

$$
K=.815 \frac{e}{m} \frac{\lambda}{\bar{c} \sqrt{\mathrm{I}+.575 \frac{V e \lambda}{m d \bar{c}^{2} \sqrt{f}}}}
$$

on the basis of Townsend's reasoning. This of course only holds for the values of a terminal energy of agitation which is less than the resonance value $R e$ discussed above. That such a fractional loss of energy $(f)$ actually may occur in nitrogen gas is shown both by the results of Franck and Hertz, ${ }^{9}$ and those of Compton. ${ }^{10}$ The mobility of the electron in the equation above varies with the voltage, the distance between the plates, and with the pressure; for $\lambda$ the mean free path varies with the pressure. The form of the variation of $K$ with these quantities for the equation would however be

$$
K=\frac{a}{\sqrt{\mathrm{r}+\frac{b V}{p d}}}
$$

while the observed equation makes

$$
K=\frac{a}{b+\frac{V}{p d}} .
$$

The two equations, observed and theoretical, may only be caused to agree in case either $\sqrt{f}$, or $\lambda$, vary as a function of $(V / p d)$. In the former case agreement can be had only if $\sqrt{f}$ increases as the value of $V / p d$ decreases. That is when the loss of energy of the electron on impact ${ }^{9}$ Franck and Hertz, Verh. d. Deut. Phys. Ges., I5, p. 34, I9r3; Verh. d. Deut. Phys. Ges., I5, p. 373 , I913.

10 Compton, K. T., Phys. Rev., Vol. XI., 3, p. 196, March, I9.18.

11 Pidduck, F. B., Proc. Roy. Soc., A 88, p. 300, r9r3.

12 Lenard, P., Ann. der Phys., Vol. 40, p. 409, I9r3. 
Vou. XIX.] MOBILITY OF ELECTRONS IN PURE NITROGEN.
No. I.

decreases as the energy of the electron increases. It is well known that such a variation of the loss of energy of the electron occurs on impacts when the electrons move with high speeds. Whether such can be the case at the low values of the velocities of the electrons dealt with here is, however, doubtful.

(d) Value of the Mean Free Path of Electrons in Nitrogen Deduced from the Results on the Basis of Townsend's Theory.-Finally if in the general experimental equation for the mobility of the electron,

$$
K=\frac{57 \mathrm{I}, 000}{2 \mathrm{I}+\frac{760 V}{p d}},
$$

the value of $V$ be set equal to o, it is seen that $K$ becomes $27,200 \mathrm{~cm} . / \mathrm{sec}$. volt $/ \mathrm{cm}$. On the Townsend point of view this represents the value of the mobility when the electron has a velocity equal to its velocity of thermal agitation in equilibrium with the gas molecules. The value of this mobility may be computed from the Townsend equation when $V$ approaches 0 , by setting the mean free path of the electrons as $4 \sqrt{2}$ that of the nitrogen molecules. The value thus obtained is $6940 \mathrm{~cm} . / \mathrm{sec}$. volt $/ \mathrm{cm}$. This marked difference in the two values may be interpreted as meaning one of two things. Either that one is not justified in applying the Langevin equation to the case of the electron at all, a point of view which may after all be correct; or that the mean free path of the electron is 3.9 times as great as that which has been assumed above. ${ }^{13}$ If the latter view point is correct it is likely that the definition of the "mean free path" of an electron in a gas must be distinctly modified.

In conclusion the writer wishes to express his thanks to Prof. R. A. Millikan for his kind advice and criticism. The writer also desires to acknowledge his indebtedness to Dr. A. J. Dempster for many valuable suggestions and criticisms related to the more theoretical aspects of this paper.

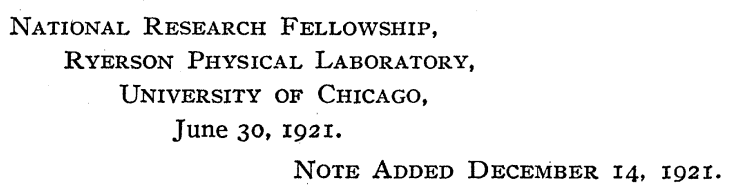

In applying these measurements to electrons in $\mathrm{H}_{2}$ gas a new oscillating system was used in which the voltage $V$ could be varied from ro200 volts without changing the frequency. Measurements made on $\mathrm{N}_{2}$ checked the values here given within the limits of experimental variation.-R. B. LOEB.

13 This high value of the mean free path may have some justification in view of the recent results of Mayer and Ramsauer on argon at velocities of electrons below 3 volts. Ann. der Phys., Vol. 64, p. 45I and p. 6r3, r92I. 